\title{
ON FACTOR RELATIONS BETWEEN WEIGHTED AND NÖRLUND MEANS
}

\author{
G. CANAN HAZAR GÜLEÇ AND MEHMET ALI SARIGÖL
}

\begin{abstract}
By $(X, Y)$, we denote the set of all sequences $\epsilon=\left(\epsilon_{n}\right)$ such that $\Sigma \epsilon_{n} a_{n}$ is summable $Y$ whenever $\Sigma a_{n}$ is summable $X$, where $X$ and $Y$ are two summability methods. In this study, we get necessary and sufficient conditions for $\epsilon \in\left(\left|N, q_{n}, u_{n}\right|_{k},\left|\bar{N}, p_{n}\right|\right)$ and $\epsilon \in\left(\left|\bar{N}, p_{n}\right|,\left|N, q_{n}, u_{n}\right|_{k}\right), k \geq 1$, using functional analytic tecniques, where $\left|\bar{N}, p_{n}\right|$ and $\left|N, q_{n}, u_{n}\right|_{k}$ are absolute weighted and Nörlund summability methods, respectively, [1], [5]. Thus, in the special case, some well known results are also deduced.
\end{abstract}

\section{Introduction}

Let $A=\left(a_{n v}\right)$ be an infinite matrix of complex numbers, $\Sigma a_{v}$ be a given infinite series with nth partial sum $s_{n}$ and $\left(u_{n}\right)$ be a sequence of nonnegative terms. Then the series $\Sigma a_{\nu}$ is called summable $\left|A, u_{n}\right|_{k}, k \geq 1$, if (see [16])

$$
\sum_{n=0}^{\infty} u_{n}^{k-1}\left|A_{n}(s)-A_{n-1}(s)\right|^{k}<\infty, A_{-1}(s)=0,
$$

where $A(s)=\left(A_{n}(s)\right)$, the $A$-transform sequence of the sequence $s=\left(s_{n}\right)$, i.e.,

$$
A_{n}(s)=\sum_{v=0}^{\infty} a_{n v} s_{v}
$$

converges for $n \geq 0$. Note that if $A$ is chosen as the Nörlund matrix (resp. $u_{n}=n$ ), then the summability $\left|A, u_{n}\right|_{k}$ reduces to the absolute Nörlund summability $\left|N, p_{n}, u_{n}\right|_{k}$ [5] (resp. the summability $\left|N, p_{n}\right|_{k}$, Borwein and Cass [2]), and also $\left|N, p_{n}\right|_{1}=\left|N, p_{n}\right|$, Mears [9]. Further, if $p_{n}=\left(\begin{array}{c}\alpha+n-1 \\ n\end{array}\right)$ and $u_{n}=n$, then the summability $\left|N, p_{n}, u_{n}\right|_{k}$ is the same as the summability $|C, \alpha|_{k}$ in Flett's notation [4]. By a Nörlund matrix we mean one that

$$
a_{n v}=\left\{\begin{array}{cc}
p_{n-v} / P_{n}, & 0 \leq v \leq n \\
0, & v>n,
\end{array}\right.
$$

Received December 27, 2017, accepted July 23, 2018.

2010 Mathematics Subject Classification. 40C05, 40D25, 40F05, 46A45.

Key words and phrases. Sequence spaces, absolute Nörlund summability, absolute weighted mean summability, summability factors.

Corresponding author: G. Canan Hazar Güleç. 
where $\left(p_{n}\right)$ is a sequence of complex numbers with $P_{n}=p_{0}+p_{1}+\cdots+p_{n} \neq 0, P_{-(n+1)}=0$ for $n \geq 0$. Also, if $A=\left(a_{n v}\right)$ is the weighted matrix (resp. $\left.u_{n}=P_{n} / p_{n}\right)$, i.e.

$$
a_{n v}=\left\{\begin{array}{c}
p_{v} / P_{n}, 0 \leq v \leq n \\
0, \quad v>n
\end{array}\right.
$$

then the summability $\left|A, u_{n}\right|_{k}$ reduces to the summability $\left|\bar{N}, p_{n}, u_{n}\right|_{k}$ (resp. the summability $\left|\bar{N}, p_{n}\right|_{k}$, Bor [1]), where $\left(p_{n}\right)$ is a sequence of positive numbers such that $P_{n}=p_{0}+p_{1}+\cdots+$ $p_{n} \rightarrow \infty$ as $n \rightarrow \infty$, Sulaiman [22]. For example, for the summability $\left|\bar{N}, p_{n}\right|_{k}$, the condition (1.1) may be stated as

$$
\sum_{n=1}^{\infty}\left|\frac{1}{P_{n-1}}\left(\frac{p_{n}}{P_{n}}\right)^{1 / k^{*}} \sum_{\nu=1}^{\infty} P_{\nu-1} a_{\nu}\right|^{k}<\infty .
$$

Throughout this paper, $k^{*}$ is the conjugate of $k>1$, i.e., $1 / k+1 / k^{*}=1$, and $1 / k^{*}=0$ for $k=1$.

For any real $\alpha$ and integers $n \geq 0$, we define

$$
\Delta^{\alpha} \epsilon_{n}=\sum_{\nu=n}^{\infty} A_{\nu-n}^{-\alpha-1} \epsilon_{v}
$$

whenever the series on right side of equality is convergent.

Let $\epsilon$ be a sequence and $X$ and $Y$ be two methods of summability. If $\Sigma \epsilon_{n} a_{n}$ is summable $Y$ whenever $\Sigma a_{n}$ is summable $X$, then $\epsilon$ is said to be a summability factor of type $(X, Y)$ and we denote it by $\epsilon \in(X, Y)[3]$. The problems of summability factors dealing with absolute Cesàro and absolute weighted mean summabilities were widely examined by many authors (see [1-4], [8-11] , [13-21]) et al. For example, for $\alpha \geq 0, k>1$, the summability factors of type $\left(|C, \alpha|,\left|\bar{N}, p_{n}\right|\right),\left(|C, \alpha|_{k},\left|\bar{N}, p_{n}\right|\right),\left(|C, \alpha|_{k},|C, 1|\right)$ and $\left(|C, 1|_{k},\left|\bar{N}, p_{n}\right|\right)$ were characterized by Mohapatra [11], Mazhar [8], Mehdi [10], Sarıgöl and Bor [17] and Sarıgöl [18], respectively. In a more recent paper, Sarıgöl [13] has extended these classes to $\alpha>-1$ and arbitrary positive sequence $\left(p_{n}\right)$ in the following form.

Theorem 1.1. Let $\alpha>-1$ and $\left(p_{n}\right)$ be arbitrary sequence of positive numbers. Then, necessary and sufficient condition for $\epsilon \in\left(|C, \alpha|_{k},\left|\bar{N}, p_{n}\right|\right), k>1$, is

$$
\sum_{m=1}^{\infty} m^{\alpha k^{*}+k^{*}-1}\left(\sum_{n=m}^{\infty} \frac{p_{n}}{P_{n} P_{n-1}}\left|\sum_{r=m}^{\infty} A_{r-m}^{-\alpha-1} \frac{\epsilon_{r}}{r} P_{r-1}\right|\right)^{k^{*}}<\infty .
$$

\section{Main results}

The purpose of this study is to generalize Theorem 1.1 by using Nörlund mean in place of Cesàro mean. Hence we characterize both classes $\left(\left|N, q_{n}, u_{n}\right|_{k},\left|\bar{N}, p_{n}\right|\right)$ and 
$\left(\left|\bar{N}, p_{n}\right|,\left|N, q_{n}, u_{n}\right|_{k}\right)$, which gives us more than we need. In the special cases, some well known results are also deduced.

Before stating the theorems we recall the following lemmas which plays important role for the proof our theorems.

Lemma 2.1. Let $1<k<\infty$. Then, $A(x) \in \ell$ whenever $x \in \ell_{k}$ if and only if

$$
\sum_{\nu=0}^{\infty}\left(\sum_{n=0}^{\infty}\left|a_{n v}\right|\right)^{k^{*}}<\infty
$$

where $\ell_{k}=\left\{x=\left(x_{v}\right): \Sigma\left|x_{v}\right|^{k}<\infty\right\}[13]$.

Lemma 2.2. Let $1 \leq k<\infty$. Then, $A(x) \in \ell_{k}$ whenever $x \in \ell$ if and only if

$$
\sup _{v} \sum_{n=0}^{\infty}\left|a_{n v}\right|^{k}<\infty,
$$

[7].

Now we begin with the theorem characterizing the class $\left(\left|N, q_{n}, u_{n}\right|_{k},\left|\bar{N}, p_{n}\right|\right)$.

Theorem 2.3. Let $q_{0}$ be a non-zero number, $\left(u_{n}\right)$ be a sequence of positive terms and $\left(C_{n}\right)$ be a sequence satisfying

$$
\sum_{\nu=0}^{n} Q_{n-\nu} C_{\nu}=\left\{\begin{array}{c}
1, n=0 \\
0, n \geq 1 .
\end{array}\right.
$$

Then necessary and sufficient condition for $\epsilon \in\left(\left|N, q_{n}, u_{n}\right|_{k},\left|\bar{N}, p_{n}\right|\right), k>1$, is

$$
\sum_{m=1}^{\infty} \frac{1}{u_{m}}\left(\sum_{n=m}^{\infty}\left|\frac{p_{n}}{P_{n} P_{n-1}} \sum_{r=m}^{n} P_{r-1} \epsilon_{r} G_{r m}\right|\right)^{k^{*}}<\infty
$$

where

$$
G_{n r}=\sum_{\nu=r}^{n} C_{n-\nu} Q_{\nu}
$$

Proof. Let $\left(t_{n}\right)$ and $\left(T_{n}\right)$ be the sequences of Nörlund mean $\left(N, q_{n}\right)$ and weighted mean $\left(\bar{N}, p_{n}\right)$ of the series $\Sigma a_{n}$ and $\Sigma \epsilon_{n} a_{n}$, respectively, i.e

$$
t_{n}=\frac{1}{Q_{n}} \sum_{v=0}^{n} q_{n-v} s_{v}=\frac{1}{Q_{n}} \sum_{v=0}^{n} Q_{n-v} a_{v}
$$

and

$$
T_{n}=\frac{1}{P_{n}} \sum_{v=0}^{n}\left(P_{n}-P_{v-1}\right) \epsilon_{v} a_{v} .
$$

Then we define sequences $y=\left(y_{n}\right)$ and $\tilde{y}=\left(\tilde{y}_{n}\right)$ by

$$
y_{n}=u_{n}^{1 / k^{*}}\left(t_{n}-t_{n-1}\right)
$$


and

$$
\tilde{y}_{n}=T_{n}-T_{n-1}=\frac{p_{n}}{P_{n} P_{n-1}} \sum_{r=1}^{n} P_{r-1} \epsilon_{r} a_{r}, n \geq 1 \text { and } \tilde{y}_{0}=a_{0} \epsilon_{0}
$$

Then, $\epsilon \in\left(\left|N, q_{n}, u_{n}\right|_{k},\left|\bar{N}, p_{n}\right|\right)$ if and only if $\tilde{y} \in l$ whenever $y \in l_{k}$. On the other hand, since $q_{0}$ is a non-zero, there exists a sequence $\left(C_{n}\right)$ satisfying $(2.1)$ and therefore it follows that

$$
t_{n}=\frac{1}{Q_{n}} \sum_{\nu=0}^{n} Q_{n-\nu} a_{v} \text { if and only if } a_{n}=\sum_{\nu=0}^{n} C_{n-v} Q_{\nu} t_{\nu} .
$$

Hence we get from (2.4),

$$
\begin{aligned}
a_{n} & =\sum_{\nu=0}^{n} C_{n-\nu} Q_{\nu} \sum_{r=0}^{v} u_{r}^{-1 / k^{*}} y_{r} \\
& =\sum_{r=0}^{n} u_{r}^{-1 / k^{*}} \sum_{\nu=r}^{n} C_{n-\nu} Q_{v} y_{r}=\sum_{r=0}^{n} u_{r}^{-1 / k^{*}} G_{n r} y_{r}
\end{aligned}
$$

where $G_{n r}$ is defined by (2.3), and so, for $n \geq 1$,

$$
\begin{aligned}
\tilde{y}_{n} & =\frac{p_{n}}{P_{n} P_{n-1}} \sum_{r=1}^{n} P_{r-1} \epsilon_{r} a_{r} \\
& =\frac{p_{n}}{P_{n} P_{n-1}} \sum_{r=1}^{n} P_{r-1} \epsilon_{r} \sum_{m=1}^{r} u_{m}^{-1 / k^{*}} G_{r m} y_{m} \\
& =\frac{p_{n}}{P_{n} P_{n-1}} \sum_{m=1}^{n}\left(u_{m}^{-1 / k^{*}} \sum_{r=m}^{n} P_{r-1} \epsilon_{r} G_{r m}\right) y_{m} \\
& =\sum_{m=1}^{n} b_{n m} y_{m}
\end{aligned}
$$

where

$$
b_{n m}= \begin{cases}\frac{u_{m}^{-1 / k^{*}} p_{n}}{P_{n} P_{n-1}} \sum_{r=m}^{n} P_{r-1} \epsilon_{r} G_{r m}, & m \leq n \\ 0, & m>n .\end{cases}
$$

Then, $\tilde{y} \in l$ whenever $y \in l_{k}$ if and only if

$$
\sum_{m=1}^{\infty}\left(\sum_{n=m}^{\infty}\left|b_{n m}\right|\right)^{k^{*}}<\infty
$$

by Lemma 2.1, which is same as the condition (2.2). This completes the proof.

It may be remarked that in the special case $q_{n}=A_{n}^{\alpha-1}$ and $u_{n}=n$, Theorem 2.3 reduces to Theorem 1.1. In fact, in this case it is obvious that $\left|N, q_{n}, u_{n}\right|_{k}=|C, \alpha|_{k}$. Also, we recall the following well known equality of Bosanquet and Das [3], for $\alpha \neq-1,-2, \ldots, v \geq 1$,

$$
\sum_{r=v}^{n} A_{r}^{\alpha} A_{n-r}^{-\alpha-2}=\frac{v A_{v}^{\alpha} A_{n-v}^{-\alpha-1}}{n},
$$


Now, it is easy to see that $C_{0}=A_{0}^{-\alpha-2}=1, C_{n}=A_{n}^{-\alpha-2}$ and $A_{-n}^{\alpha}=0$ for $n \geq 1$, and so, since

$$
G_{r m}=\sum_{v=m}^{r} A_{v}^{\alpha} A_{r-v}^{-\alpha-2}=\frac{m A_{m}^{\alpha} A_{r-m}^{-\alpha-1}}{r}, 1 \leq r \leq v \text {, and } 0 \text { for } r>v \text {, }
$$

by using (2.6), we get the matrix $B=\left(b_{n m}\right)$ as

$$
b_{n m}= \begin{cases}\frac{m^{1 / k} A_{m}^{\alpha} p_{n}}{P_{n} P_{n-1}} \sum_{r=m}^{n} A_{r-m}^{-\alpha-1} \frac{\epsilon_{r}}{r} P_{r-1}, & m \leq n \\ 0, & m>n .\end{cases}
$$

So by $A_{n}^{\alpha} \sim n^{\alpha} / \Gamma(\alpha+1)$ for $\alpha>-1$ [4], it follows from applying Lemma 2.1 to the matrix $B$ that (2.2) is the same as (1.4), as asserted.

Note that $1 \in(X, Y)$ leads us to a comparison of summability fields of methods $X$ and $Y$, where $1=(1,1, \ldots)$. So taking $\epsilon_{n}=u_{n}=1$ for all $n \geq 1$ in Theorem 2.3 we get the following result.

Corollary 2.4. If $k>1$, then, $1 \in\left(\left|N, q_{n}\right|_{k},\left|\bar{N}, p_{n}\right|\right)$ if and only if

$$
\sum_{m=1}^{\infty}\left(\sum_{n=m}^{\infty}\left|\frac{p_{n}}{P_{n} P_{n-1}} \sum_{r=m}^{n} P_{r-1} G_{r m}\right|\right)^{k^{*}}<\infty
$$

This result also extends the following result of Kayashima [6] to $k>1$.

Corollary 2.5. If $\left(p_{n}\right)$ and $\left(q_{n}\right)$ are positive and nonincreasing sequences and $\left(q_{n+1} / q_{n}\right)$ is nondecreasing, then $1 \in\left(\left|N, q_{n}\right|,\left|\bar{N}, p_{n}\right|\right)$.

Theorem 2.6. Let $k \geq 1$ and $\left(u_{n}\right)$ be a sequence of nonnegative terms. Then, $\epsilon \in\left(\left|\bar{N}, p_{n}\right|,\left|N, q_{n}, u_{n}\right|_{k}\right)$ if and only if

$$
\sup _{v}\left\{u_{v}^{1 / k^{*}}\left|\frac{\epsilon_{v} P_{v}}{Q_{v} p_{v}}\right|\right\}<\infty
$$

and

$$
\sup _{v} \sum_{n=v+1}^{\infty}\left|u_{n}^{1 / k^{*}}\left(\Omega_{n v}^{q} \epsilon_{v} \frac{P_{v}}{p_{v}}-\Omega_{n, v+1}^{q} \epsilon_{v+1} \frac{P_{v-1}}{p_{v}}\right)\right|^{k}<\infty,
$$

where

$$
\Omega_{n v}^{q}=\frac{Q_{n-v}}{Q_{n}}-\frac{Q_{n-v-1}}{Q_{n-1}}, Q_{-1}=0 .
$$

Proof. As in proof of Theorem 2.3, we define sequences $y=\left(y_{n}\right)$ and $\tilde{y}=\left(\tilde{y}_{n}\right)$ by $y_{0}=\epsilon_{0} a_{0}$,

$$
y_{n}=u_{n}^{1 / k^{*}}\left(t_{n}-t_{n-1}\right)=u_{n}^{1 / k^{*}} \sum_{v=0}^{n}\left(\frac{Q_{n-v}}{Q_{n}}-\frac{Q_{n-v-1}}{Q_{n-1}}\right) \epsilon_{v} a_{v}, n \geq 1
$$




$$
\tilde{y}_{0}=a_{0}, \tilde{y}_{n}=T_{n}-T_{n-1}=\frac{p_{n}}{P_{n} P_{n-1}} \sum_{r=1}^{n} P_{r-1} a_{r}, n \geq 1
$$

Then, $\epsilon \in\left(\left|\bar{N}, p_{n}\right|,\left|N, q_{n}, u_{n}\right|_{k}\right)$ iff $y \in l_{k}$ whenever $\tilde{y} \in l$. On the other hand, from (2.12) we write

$$
a_{n}=\frac{P_{n}}{p_{n}} \tilde{y}_{n}-\frac{P_{n-2}}{p_{n-1}} \tilde{y}_{n-1}, n \geq 1 \text { and } a_{0}=\tilde{y}_{0}
$$

Hence, by (2.11) we get

$$
\begin{aligned}
y_{n} & =u_{n}^{1 / k^{*}} \sum_{v=1}^{n} \Omega_{n v}^{q} \epsilon_{v} a_{v}=u_{n}^{1 / k^{*}} \sum_{v=1}^{n} \Omega_{n v}^{q} \epsilon_{v}\left(\frac{P_{v}}{p_{v}} \tilde{y}_{v}-\frac{P_{v-2}}{p_{v-1}} \tilde{y}_{v-1}\right) \\
& =u_{n}^{1 / k^{*}}\left\{\Omega_{n n}^{q} \epsilon_{n} \frac{P_{n}}{p_{n}} \tilde{y}_{n}+\sum_{v=1}^{n-1}\left(\Omega_{n v}^{q} \epsilon_{v} \frac{P_{v}}{p_{v}}-\Omega_{n, v+1}^{q} \epsilon_{v+1} \frac{P_{v-1}}{p_{v}}\right) \tilde{y}_{v}\right\} \\
& =\sum_{v=1}^{n} c_{n v} \tilde{y}_{v}
\end{aligned}
$$

where

$$
c_{n v}= \begin{cases}u_{n}^{1 / k^{*}}\left(\Omega_{n v}^{q} \epsilon_{v} \frac{P_{v}}{p_{v}}-\Omega_{n, v+1}^{q} \epsilon_{v+1} \frac{P_{v-1}}{p_{v}}\right), & 1 \leq v \leq n-1 \\ u_{n}^{1 / k^{*}} \Omega_{n n}^{q} \epsilon_{n} \frac{P_{n}}{p_{n}}, & v=n \\ 0, & v>n .\end{cases}
$$

So $y \in l_{k}$ whenever $\tilde{y} \in l$ if and only if

$$
\sup _{v} \sum_{n=v}^{\infty}\left|c_{n v}\right|^{k}<\infty
$$

by Lemma 2.2 or, equivalently,

$$
\sup _{v}\left\{u_{v}^{1 / k^{*}}\left|\frac{\epsilon_{v} P_{v}}{Q_{v} p_{v}}\right|\right\}<\infty, v \geq 1
$$

and

$$
\sup _{v} \sum_{n=v+1}^{\infty}\left|u_{n}^{1 / k^{*}}\left(\Omega_{n v}^{q} \epsilon_{v} \frac{P_{v}}{p_{v}}-\Omega_{n, v+1}^{q} \epsilon_{v+1} \frac{P_{v-1}}{p_{v}}\right)\right|^{k}<\infty .
$$

Thus the proof is completed.

Corollary 2.7. If $k \geq 1$, then, $1 \in\left(\left|\bar{N}, p_{n}\right|,\left|N, q_{n}\right|_{k}\right)$ if and only if

$$
\sup _{v}\left\{v^{1 / k^{*}}\left|\frac{P_{v}}{Q_{v} p_{v}}\right|\right\}<\infty
$$

and

$$
\sup _{v} \sum_{n=v+1}^{\infty}\left|n^{1 / k^{*}}\left(\Omega_{n v}^{q} \frac{P_{v}}{p_{v}}-\Omega_{n, v+1}^{q} \frac{P_{v-1}}{p_{v}}\right)\right|^{k}<\infty .
$$


Proof. Put $\epsilon_{n}=1$ and $u_{n}=n$ for all $n \geq 1$ in Theorem 2.6.

This result, for $k=1$, reduces to the following theorem of Kayashima [6].

Corollary 2.8. If $\left(p_{n}\right)$ and $\left(q_{n}\right)$ are positive and nondecreasing sequences and $\left(q_{n+1} / q_{n}\right)$ is nonincreasing, then $1 \in\left(\left|\bar{N}, p_{n}\right|,\left|N, q_{n}\right|\right)$.

Proof. By considering that $\left(p_{n}\right)$ and $\left(q_{n}\right)$ are positive and nondecreasing sequences, we have

$$
\frac{P_{n}}{Q_{n} p_{n}} \leq \frac{n+1}{Q_{n}} \leq q_{0}^{-1} \text { for all } n \geq 0 .
$$

Also, by hypotheses on the sequence $\left(q_{n}\right)$, it converges to a number, $\lim _{n} q_{n+1} / q_{n}=\sigma$ say. So, there exists a nonincreasing null sequence $\left(x_{n}\right)$ such that $q_{n+1}=\left(\sigma+x_{n}\right) q_{n}$ for all $n \geq 0$, where $\sigma \geq 1$. Then, it can be written that

$$
Q_{n}=q_{0}+\sigma Q_{n-1}+\sum_{v=1}^{n} q_{v-1} x_{v-1}
$$

which gives

$$
\frac{Q_{n}}{Q_{n-1}}=\frac{q_{0}}{Q_{n-1}}+\sigma+Z_{n} \rightarrow \sigma \text { as } n \rightarrow \infty
$$

where

$$
Z_{n}=\frac{1}{Q_{n-1}} \sum_{v=1}^{n} q_{v-1} x_{v-1}
$$

Since $\left(x_{n}\right)$ is nonincreasing, it is easily seen that $\left(Z_{n}\right)$ is nonincreasing, which implies that $\left(Q_{n} / Q_{n-1}\right)$ is nonincreasing. So it follows that, for $0 \leq v \leq n$,

$$
\Omega_{n v}^{q}=\frac{Q_{n-v-1}}{Q_{n}}\left(\frac{Q_{n-v}}{Q_{n-v-1}}-\frac{Q_{n}}{Q_{n-1}}\right) \geq 0
$$

Further,

$$
C_{n v}=\Omega_{n v}^{q} \frac{P_{v}}{p_{v}}-\Omega_{n, v+1}^{q} \frac{P_{v-1}}{p_{v}} \geq 0 .
$$

In fact, if $q_{n-v} / Q_{n}-q_{n-v-1} / Q_{n-1} \geq 0$, then it is clear that $C_{n v} \geq 0$, since

$$
C_{n v}=\frac{P_{v}}{p_{v}}\left(\frac{q_{n-v}}{Q_{n}}-\frac{q_{n-v-1}}{Q_{n-1}}\right)+\Omega_{n, v+1}^{q} .
$$

If $q_{n-v} / Q_{n}-q_{n-\nu-1} / Q_{n-1}<0$, then, it can be deduced from the condition on $\left(q_{n}\right)$ that

$$
\begin{aligned}
\frac{q_{n-v}}{Q_{n}}-\frac{q_{n-\nu-1}}{Q_{n-1}} & \geq \frac{q_{n-\nu-1}}{Q_{n}}\left(\frac{q_{n-m}}{q_{n-m-1}}-\frac{Q_{n}}{Q_{n-1}}\right) \\
& \geq \frac{q_{n-m}}{Q_{n}}-\frac{q_{n-m-1}}{Q_{n-1}}, 0 \leq m \leq \nu,
\end{aligned}
$$


which implies

$$
v\left(\frac{q_{n-v}}{Q_{n}}-\frac{q_{n-v-1}}{Q_{n-1}}\right) \geq \sum_{m=0}^{v-1}\left(\frac{q_{n-m}}{Q_{n}}-\frac{q_{n-m-1}}{Q_{n-1}}\right)=-\Omega_{n v}^{q} .
$$

Also, since $\left(p_{\nu}\right)$ is a positive nondecreasing sequence, we can write $P_{v} \leq v p_{v}$ for all $v \geq 1$, which gives us, by (2.17),

$$
\frac{P_{v}}{p_{v}}\left(\frac{q_{n-v}}{Q_{n}}-\frac{q_{n-v-1}}{Q_{n-1}}\right) \geq-\frac{P_{v}}{v p_{v}} \Omega_{n v}^{q} \geq-\Omega_{n v}^{q} .
$$

This means that $C_{n v} \geq 0$ for $0 \leq v \leq n$. Hence, by considering (2.15), we have

$$
\begin{aligned}
\sup _{v} \sum_{n=v+1}^{\infty}\left|\frac{P_{v}}{p_{v}} \Omega_{n v}^{q}-\frac{P_{v-1}}{p_{v}} \Omega_{n, v+1}^{q}\right| & =\sup _{v} \lim _{m} \sum_{n=v+1}^{m}\left(\frac{P_{v}}{p_{v}} \Omega_{n v}^{q}-\frac{P_{v-1}}{p_{v}} \Omega_{n, v+1}^{q}\right) \\
& \leq \sup _{v} \lim _{m}\left[\frac{P_{v}}{p_{v}}\left(\frac{Q_{m-v}}{Q_{m}}-\frac{q_{0}}{Q_{v}}\right)-\frac{P_{v-1} Q_{m-v-1}}{p_{v} Q_{m}}\right] \\
& =\sup _{v}\left[\frac{P_{v}}{p_{v}}\left(\frac{1}{\sigma^{v}}-\frac{q_{0}}{Q_{v}}\right)-\frac{P_{\nu-1}}{p_{v} \sigma^{\nu-1}}\right]<\infty,
\end{aligned}
$$

which completes the proof.

Further the following result of [12] is obtained form Corollary 2.7 by choosing $q_{n}=1$ for $n \geq 1$.

Corollary 2.9. If $k \geq 1$, then, $1 \in\left(\left|\bar{N}, p_{n}\right|,|C, 1|_{k}\right)$ if and only if

$$
\sup _{v} \frac{P_{v}}{v^{1 / k} p_{v}}<\infty
$$

\section{References}

[1] H. Bor, On two summability methods, Math. Proc. Cambridge Philos. Soc., 97 (1985), No.1, 147-149.

[2] D. Borwein and F. P. Cass, Strong Nörlund summability, Math. Zeitschr., 103 (1968), 94-111.

[3] L. S. Bosanquet and G. Das, Absolute summability factors for Nörlund means, Proc. London Math. Soc. (3), 38 (1979), 1-52.

[4] T. M. Flett, On an extension of absolute summability and some theorems of Littlewood and Paley, Proc. London Math. Soc., 7 (1957), 113-141.

[5] G. C. Hazar and M.A Sarıgöl, On absolute Nörlund spaces and matrix operators, Acta Math. Sin. (Engl. Ser.), (2018), 34 (5), 812-826.

[6] I. Kayashima, On relations between Nörlund and Riesz means, Pac. J. Math., 49 (2) (1973), 391-396.

[7] I. J. Maddox, Elements of Functional Analysis, Cambridge University Press, London,New York, 1970.

[8] S. M. Mazhar, On the absolute summability factors of infinite series, Tohoku Math. J., 23 (1971), 433-451.

[9] M. F. Mears, Absolute Regularity and the Nörlund Mean, Annals of Math., 38 (3), (1937), 594-601.

[10] M. R. Mehdi, Summability factors for generalized absolute summability I, Proc. London Math. Soc. (3), 10 (1960), 180-199.

[11] R. N. Mohapatra, On absolute Riesz summability factors, J. Indian Math. Soc., 32 (1968), 113-129. 
[12] C. Orhan and M. A. Sarıgöl, On absolute weighted mean summability, Rocky Mount. J. Math., 23 (1993), 1091-1097.

[13] M. A. Sarıgöl, Extension of Mazhar's theorem on summability factors, Kuwait J. Sci., 42 (3) (2015), $28-35$.

[14] M. A. Sarıöl, Matrix operators on $A_{k}$, Math. Comp. Model., 55 (2012), 1763-1769.

[15] M. A. Sarıgöl, Matrix transformations on fields of absolute weighted mean summability, Studia Sci. Math. Hungar., 48 (3) (2011), 331-341.

[16] M. A. Sarıöl, On the local properties of factored Fourier series, Appl. Math. Comp., 216 (2010), 3386-3390.

[17] M. A. Sarıgöl and H. Bor, Characterization of absolute summability factors, J. Math. Anal. Appl., 195 (1995), 537-545.

[18] M. A. Sarıöl, On two absolute Riesz summability factors of infinite series, Proc. Amer. Math. Soc., 118 (1993), 485-488.

[19] M. A. Sarı̈öl, A note on summability, Studia Sci. Math. Hungar., 28 (1993), 395-400.

[20] M. A. Sarı̈öl, On absolute weighted mean summability methods, Proc. Amer. Math. Soc., 115 (1) (1992), $157-$ 160.

[21] M.A. Sarı̈öl, Necessary and sufficient conditions for the equivalence of the summability methods $\left|\bar{N}, p_{n}\right|_{k}$ and $|C, 1|_{k}$, Indian J. Pure Appl. Math., 22 (6) (1991), 483-489.

[22] W. T. Sulaiman, On summability factors of infinite series, Proc. Amer. Math. Soc., 115 (1992), 313-317.

Department of Mathematics, Pamukkale University, TR-20007 Denizli, Turkey.

E-mail: gchazar@pau.edu.tr

Department of Mathematics, Pamukkale University, TR-20007 Denizli, Turkey.

E-mail: msarigol@pau.edu.tr 